\title{
Relación entre diabetes gestacional y síndrome metabólico
}

\author{
Cecilia Ferradala, Marta Molinala, Luis Cid²b, \\ Gisela Riedel ${ }^{3}$, Cristina Ferrada ${ }^{1 a}$, Rodrigo Arévalo ${ }^{4}$ \\ Relationship between gestational \\ diabetes and metabolic syndrome
}

Background: The metabolic syndrome (MS) is associated to a significant increase in the risk of diabetes, coronary heart disease and stroke, resulting in a 5fold increase of the cardiovascular death rate. Aim: To determine the relationship between gestational diabetes (GD) and the development of MS at the end of puerperal period. Patients and Methods: We conducted a case-control study in the Curanilahue Hospital, in southern Chile, including 58 women with GD during their pregnancy, studied in day 42 of their puerperal period (study group) and 58 puerperal women from the same hospital, who had a physiological pregnancy (control group). Triglycerides, HDL cholesterol, fasting and post prandial blood glucose levels, blood pressure, waist circumference, weight and height were measured. MS was diagnosed using the National Cholesterol Education Program (NCEP - ATP III) criteria. Results: Mean weight, body mass index, waist circumference, blood glucose and triglycerides were significantly higher in the study group and HDL cholesterol was significantly lower in the control group. Conclusions: These results suggest a significant relationship between GD and the ocurrence of MS (Rev Méd Chile 2007; 135: 1539-45).

(Key words: Diabetes, gestational; Insulin resistance; Pregnancy complications)
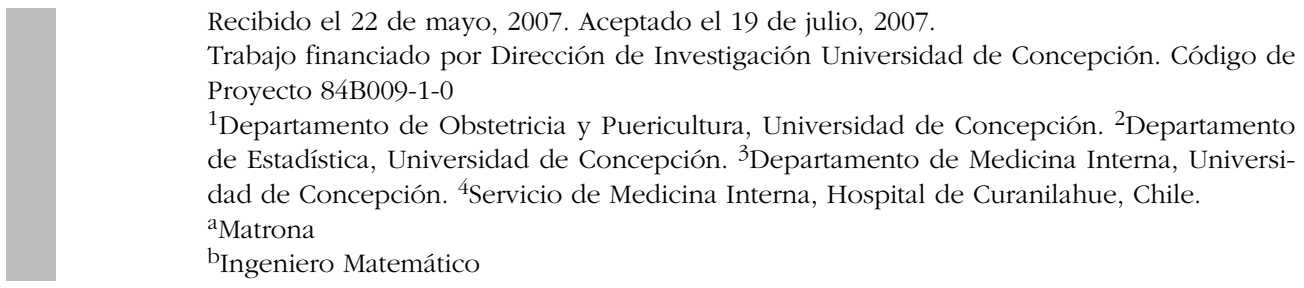

$\mathrm{E}^{1}$ síndrome metabólico es una asociación de problemas de salud que pueden aparecer de forma simultánea o secuencial en un mismo individuo, causado por una combinación de facto-

Correspondencia a: Marta Molina S. Departamento de Obstetricia y Puericultura, Universidad de Concepción, Concepción. E mail: marmolin@udec.cl res genéticos y ambientales asociados al estilo de vida, en los que la resistencia a la insulina se considera el componente patogénico fundamen$\mathrm{tal}^{1}$.

Según el NCEP-ATP III $^{2}$, los criterios para el diagnóstico del SM incluyen la obesidad abdominal (reflejada en circunferencia cintura), triglicéridos séricos elevados, colesterol HDL bajo, presión arterial alta o tratamiento antihipertensivo y nive- 
les elevados de glicemia. Entre los factores de riesgo asociados al SM, la resistencia a la insulina desempeña un papel central en el desarrollo de la diabetes tipo 2, la hipertensión y la enfermedad arterial cardiovascular

Aproximadamente entre $10 \%$ y $30 \%$ de la población mundial padece de SM. La prevalencia de SM en Chile es equivalente a la de Estados Unidos de Norteamérica (EE.UU.) poniendo una vOz de alerta sobre el aumento de riesgo cardiovascular ${ }^{3}$.

En EE.UU., la prevalencia del SM en mayores de 20 años es de $24 \%$, en mayores de 50 años es alrededor de 30\%, y 40\% después de los 60 años. Hay una leve mayor prevalencia entre mujeres $(24,7 \%$ vs. $21,7 \%)$ y población caucásica con $24,1 \%$ versus $19.3 \%$ en afroamericanos y $16,5 \%$ en otras razas ${ }^{4}$

En Chile, la prevalencia del SM en adultos es de $22,6 \% ; 23 \%$ en hombres y $22,3 \%$ en mujeres. En rangos de 17 a 24 años la prevalencia es de 4,6\%; 25 a 44 años, 17,9\%; 45 y 64 años, 36,5\% y $48 \%$ en mayores de 64 años 5 .

En poblaciones de alto riesgo, como la de familiares de personas con diabetes, la prevalencia aumenta a casi 50\%, llegando a más de $80 \%$ en personas diabéticas ${ }^{3}$.

Entre las consideraciones patogénicas del SM, la insulinorresistencia se considera como la responsable de la mayor parte de las anomalías presentes en éste; la hiperglicemia, la hipertensión, el aumento en la producción hepática de VLDL y triglicéridos, y la estimulación en la proliferación endotelial causante del inicio del proceso de aterosclerosis 6 .

\section{Síndrome Metabólico y Diabetes Gestacional}

La diabetes gestacional (DG) es una alteración del metabolismo de los hidratos de carbono de severidad variable, que se inicia durante el embarazo. En Chile, se estima una prevalencia entre 3 y $5 \%$, aumentando a 10-14\% al considerar las embarazadas con factores de riesgo diabético como antecedentes familiares, edad materna sobre 30 años, obesidad, DG en embarazos anteriores, entre otros ${ }^{7}$. A nivel socio económico medio-bajo, las mujeres presentan una alta incidencia de DG, asociada a una alta prevalencia de sobrepeso y obesidad en la etapa pregestacional $(37,8 \%)^{7}$.
Masuzaki y Paterson ${ }^{8}$ reportan que el índice de masa corporal (IMC), el peso, los antecedentes familiares de diabetes mellitus tipo 2 y edad han demostrado ser factores de riesgo para SM. La frecuencia levemente elevada, aunque no significativa, de hipertensión en las mujeres con DG, concuerda con el estado de insulinorresistencia gatillado durante el embarazo y apoya la idea de DG como modelo de diabetes mellitus tipo 2. La relación directa de la edad con el desarrollo de DG, puede reflejar la existencia de otros factores de riesgo, que requieren tiempo para expresarse y desarrollar insulinorresistencia.

Jensen et al.,9 indican que las pacientes con hiperglicemia anterior al embarazo presentaron un patrón metabólico alterado con respecto a las mujeres con glicemias normales, con valores más altos de IMC, presión arterial, glicemia en ayunas, insulinemia y niveles más bajos de colesterol HDL. El predominio del SM y de sus componentes en mujeres con hiperglicemia anterior al embarazo, es 10 veces más alto si existió obesidad antes de la gestación. Concluyendo que en mujeres jóvenes adultas, la hiperglicemia anterior al embarazo, conjuntamente con obesidad, anticipan el diagnóstico de SM.

Isomaa y Henricsson, ${ }^{10}$ estudiaron la asociación del SM con resistencia a la insulina, tolerancia anormal a la glucosa, dislipidemias, obesidad e hipertensión arterial, en dos grupos de mujeres: 21 mujeres que presentaron DG y otro grupo de 18 mujeres que presentaron un embarazo fisiológico. Los análisis multivariables revelaron diferencias significativas con relación a la insulinorresistencia y variables asociadas a SM como glicemia, triglicéridos, presión arterial e IMC. Las mujeres con historia de DG presentaron las variables asociadas a SM, siendo esto atribuible a la resistencia a la insulina.

Tiikkainen et al., ${ }^{11}$ en un estudio para comparar variables asociadas a la resistencia insulínica en pacientes con DG y embarazadas no diabéticas, demostraron que muchos de los componentes metabólicos asociados a SM son causantes de DG. Estos resultados reafirman la hipótesis que la DG es una fase del SM. Los investigadores sugieren que considerar DG como componente del SM proporciona un modelo para el estudio y la prevención de diabetes mellitus en grupos de edades jóvenes. 
En los últimos años, uno de los tópicos más novedosos en endocrinología, ha sido la inesperada relación entre el bajo peso de nacimiento (BPN) y las alteraciones metabólicas asociadas a mayor riesgo cardiovascular en la edad adulta ${ }^{12}$.

Estudios epidemiológicos retrospectivos originales, realizados en sujetos en la sexta década de vida, demostraron que las personas con BPN tendrían mayor predisposición a desarrollar hipertensión arterial, diabetes mellitus tipo 2, dislipidemia y obesidad central, configurando el llamado SM o síndrome $\mathrm{X}^{13}$.

Este estudio tiene como objetivo la confirmación y cuantificación de la relación entre DG y SM al término del puerperio, mediante la cuantificación del perfil bioquímico y los antecedentes perinatales y obstétricos de las pacientes, asociados con los diagnósticos de DG y SM. En efecto, la rápida desaparición del lactógeno placentario y los bajos niveles de la hormona de crecimiento después del parto llevan a una relativa deficiencia de factores anti-insulínicos, fenómeno que se revierte al final de este período.

\section{PACIENTES Y MÉTODO}

Se realizó un estudio analítico de corte transversal, tipo caso-control, en el período comprendido entre el 1 de julio y el 31 de diciembre de 2005, en el Hospital de Curanilahue. Se analizaron 58 puérperas de 42 días, que durante su embarazo presentaron DG (grupo estudio) y 58 puérperas de 42 días que no presentaron DG (grupo control), que presentaron un embarazo fisiológico, seleccionadas al azar entre pacientes del mismo hospital, mediante un procedimiento de muestreo que seleccionó sistemáticamente una de cada dos pacientes, la primera de las cuales se seleccionó al azar, asegurando así la ausencia de sesgo en la selección de la pacientes.

Se midieron los niveles de triglicéridos séricos, colesterol HDL, glicemias en ayunas y 2 horas post carga con 75 gramos de glucosa oral, presión arterial, circunferencia de cintura, peso, talla y cálculo del índice de masa corporal (IMC) de las puérperas y de sus padres, con técnicas de medición debidamente estandarizadas. ParalelaTabla 1. Comparación de promedios de variables cuantitativas,
entre los grupos estudio y control, mediante test t-Student

\begin{tabular}{|lccccc|}
\hline Grupo & \multicolumn{2}{c}{ Estudio } & \multicolumn{3}{c|}{ Control } \\
\hline Variable & Media & Varianza & Media & Varianza & t-Student \\
\hline Edad & 27.20 & 53,32 & 26.44 & 57.72 & $0,578 \mathrm{NS}$ \\
Peso & 75.51 & 127,09 & 66.18 & 83.35 & $4,937^{* *}$ \\
Talla & 155.65 & 11,94 & 154.82 & 16.95 & $1,135 \mathrm{NS}$ \\
IMC & 30.93 & 17,60 & 27.41 & 11.73 & $4,924^{* *}$ \\
CC & 92,79 & 96,06 & 81,18 & 44,22 & $7,461^{* *}$ \\
PA sistólica & 116,43 & 87,54 & 114,79 & 69,88 & $1,032 \mathrm{NS}$ \\
PA diastólica & 73,72 & 43,43 & 71,03 & 51,82 & $2,107^{*}$ \\
IMC padre & 33,03 & 6,76 & 30,91 & 3,90 & $4,854^{* *}$ \\
IMC madre & 30,60 & 6,83 & 28,01 & 3,91 & $6,020^{* *}$ \\
Peso RN & 3780,52 & 241324,29 & 3551,38 & 138612,10 & $2,832^{* *}$ \\
Talla RN & 51,03 & 2,41 & 51,29 & 3,85 & 0,612 NS \\
Glicemia & 97,44 & 115,69 & 85,77 & 125,75 & $5,736^{* *}$ \\
Glicemia p.c. & 98,70 & 545,85 & 87,01 & 231,17 & $3,197^{* *}$ \\
Triglicéridos & 159,70 & 2590,39 & 98,05 & 1260,89 & $7,572^{* *}$ \\
HDL & 36,91 & 35,97 & 46,60 & 71,22 & $7,138^{* *}$ \\
\hline
\end{tabular}

*valor-p $<0,05 ; * *$ valor-p<0,01

$I M C$ : índice de masa corporal, $C C$ : circunferencia de cintura, $P A$ : presión arterial, $R N$ : recién nacido, p.C.: post carga, HDL: high density lipoproteins. 
mente, se realizó el diagnóstico de SM de acuerdo a las normas del NCEP-ATP III.

Para la recolección de datos personales, obstétricos, factores de riesgo y mediciones diseñadas de acuerdo a los objetivos de la investigación, se aplicó una encuesta y los resultados fueron ingresados a una planilla de cálculo Excel 7.0 para su posterior análisis y determinación de la asociación entre variables.

Estadística: para determinar la validez de la información muestral, se estimaron los tamaños de muestra mínimos para obtener una precisión de al menos e $=5 \%$, con un nivel de confianza del $95 \%$. Para comparar los promedios de las variables en estudio se utilizó la prueba t de Student. En el caso de las variables cualitativas se trabajó con las frecuencias y se utilizó la prueba Chi-Cuadrado. Se consideraron como significativos valores-p $<0,01$ (altamente significativo) y $<0,05$ (significativo). Para cuantificar la relación entre DG y SM se obtuvieron las razones de riesgos (RR u odds ratio) a partir de las respectivas tablas de contingencia.

\section{Resultados}

Un análisis de los resultados globales (Tabla 1) indica que los promedios de las variables peso, IMC, obesidad abdominal, IMC del padre, IMC de la madre, peso del recién nacido (RN), glicemia en ayunas, glicemia post carga, triglicéridos y colesterol HDL, fueron significativamente mayores en el grupo estudio ( $\mathrm{p}<0,01)$.

Respecto de las variables cualitativas, la Tabla 2 muestra que la frecuencia de RN adecuado para la edad gestacional (AEG) del grupo estudio fue muy significativamente menor que en el caso del grupo control. La Tabla 3 muestra que la frecuencia de pacientes con antecedentes de DG fue muy significativamente superior que en el grupo control.

La Tabla 4 muestra que la frecuencia de antecedentes familiares de diabetes del grupo estudio fue muy significativamente mayor que en grupo control.

La Tabla 5 muestra la frecuencia de la condición del RN de los grupos estudio y control. La proporción de $\mathrm{RN}$ grande para la edad gestacional (GEG) fue significativamente mayor $(p<0,01)$ en
Tabla 2. Tabla de contingencia de la condición al nacer de las puérperas separadas por grupos estudio y control

\begin{tabular}{|lccc|}
\hline & $\begin{array}{c}\text { Pequeño } \\
\text { para la edad } \\
\text { gestacional } \\
\text { (PEG) }\end{array}$ & $\begin{array}{c}\text { Adecuado } \\
\text { para la edad } \\
\text { gestacional } \\
\text { (AEG) }\end{array}$ & Total \\
\hline Estudio & 15 & 43 & 58 \\
Control & 1 & 57 & 58 \\
Total & 16 & 100 & 116 \\
\hline
\end{tabular}

$\mathrm{CHI}^{2}: 14,210$ significativo $(\mathrm{p}<0,01)$.

Tabla 3. Tabla de contingencia para antecedentes de diabetes gestacional de las puérperas separadas por grupo estudio y control

\begin{tabular}{|lccc|}
\hline Frecuencia & $\begin{array}{c}\text { Sin } \\
\text { antecedentes }\end{array}$ & $\begin{array}{c}\text { Con } \\
\text { antecedentes }\end{array}$ & Total \\
\hline Control & 58 & 0 & 58 \\
Estudio & 36 & 22 & 58 \\
Total & 94 & 22 & 116 \\
\hline
\end{tabular}

$\mathrm{CHI}^{2}: 27,149$ significativo $(\mathrm{p}<0,01)$.

Tabla 4. Tabla de contingencia para antecedentes familiares de diabetes para grupos estudio y control

\begin{tabular}{|lccc|}
\hline Frecuencia & $\begin{array}{c}\text { Sin } \\
\text { antecedentes }\end{array}$ & $\begin{array}{c}\text { Con } \\
\text { antecedentes }\end{array}$ & Total \\
\hline Control & 50 & 7 & 57 \\
Estudio & 27 & 31 & 58 \\
Total & 77 & 38 & 115 \\
\hline
\end{tabular}

$\mathrm{CHI}^{2}:$ 22,021 significativo $(\mathrm{p}<0,01)$.

Tabla 5. Tabla de contingencia para condición del RN al nacer para grupos estudio y control

\begin{tabular}{|lccrr|}
\hline Frecuencia & AEG & PEG & GEG & Total \\
\hline Control & 53 & 3 & 2 & 58 \\
Estudio & 40 & 3 & 15 & 58 \\
Total & 93 & 6 & 17 & 116 \\
\hline
\end{tabular}

$\mathrm{CHI}^{2}: 11,758$ significativo $(\mathrm{p}<0,01)$. 
Tabla 6. Comparación de promedios (media) de variables cuantitativas, entre los grupos estudio y control, mediante test $t$-student para subgrupo de pacientes con antecedentes familiares de diabetes

\begin{tabular}{|lccccccc|}
\hline & \multicolumn{4}{c}{ Estudio } & \multicolumn{3}{c|}{ Control } \\
\hline & $\mathrm{N}$ & Media & Varianza & $\mathrm{N}$ & Media & Varianza & t-Student \\
\hline Edad & 31 & 27,32 & 55,62 & 7 & 24,14 & 59,14 & $1,014 \mathrm{NS}$ \\
Peso & 31 & 77,22 & 153,78 & 7 & 64,85 & 88,47 & $2,473^{*}$ \\
Talla & 31 & 155,22 & 7,78 & 7 & 156,00 & 47,00 & $0,493 \mathrm{NS}$ \\
IMC & 31 & 31,77 & 21,79 & 7 & 26,07 & 4,95 & $3,126^{* *}$ \\
CC & 31 & 95,67 & 133,75 & 7 & 80,14 & 48,47 & $3,394^{* *}$ \\
PA Sistólica & 31 & 119,25 & 89,86 & 7 & 117,14 & 123,80 & $0,516 \mathrm{NS}$ \\
PA Diastólica & 31 & 74,83 & 35,67 & 7 & 66,57 & 39,61 & $3,275^{* *}$ \\
IMC padre & 29 & 33,72 & 8,20 & 7 & 31,42 & 1,61 & $2,059^{*}$ \\
IMC madre & 31 & 31,61 & 8,37 & 7 & 27,42 & 1,28 & $3,735^{* *}$ \\
Peso RN & 31 & 3814,19 & 248438,49 & 7 & 3464,29 & 256595,24 & $1,673 \mathrm{NS}$ \\
Talla RN & 31 & 51,06 & 3,19 & 7 & 51,57 & 3,95 & $0,006 \mathrm{NS}$ \\
Glicemia ayunas & 31 & 100,16 & 120,53 & 7 & 86,57 & 371,28 & $2,549^{*}$ \\
glicemia postcarga & 31 & 103,16 & 530,13 & 7 & 96,42 & 457,61 & $0,708 \mathrm{NS}$ \\
TG & 31 & 175,64 & 3190,77 & 7 & 118,14 & 1801,14 & $2,526^{*}$ \\
HDL & 31 & 37,35 & 37,10 & 7 & 43,42 & 74,95 & $2,202^{*}$ \\
\hline
\end{tabular}

${ }^{*} \mathrm{p}<0,05 ; * * \mathrm{p}<0,01$

$I M C$ : índice de masa corporal, $C C$ : circunferencia de cintura, $P A$ : presión arterial, $R N$ : recién nacido, $T G$ : triglicéridos, $H D L$ : high density lipoproteins.

el grupo estudio que en los controles. Es destacable, Tabla 2, que las puérperas con DG fueron al nacer, en una importante proporción, RN pequeños para la edad gestacional (PEG). En efecto, la RR fue de 19,9, lo que indica que es aproximadamente 20 veces más probable que una puérpera que presentó la condición PEG al nacer desarrolle DG, que una que no presentó esa condición.

La Tabla 6 muestra los resultados de la comparación de los promedios del subgrupo de pacientes con antecedentes familiares de diabetes con las variables de los grupos estudio y control, encontramos diferencias significativas $(p<0,05)$ entre los promedios de ambos grupos para las siguientes variables: peso, IMC padre, glicemia en ayunas, triglicéridos y colesterol.

La Tabla 7 muestra la frecuencia de diagnóstico de SM del grupo estudio y control, al comparar ambos grupos se observó que la proporción de SM fue significativamente mayor $(\mathrm{p}<0,01)$ en el grupo de estudio. EL valor calculado para la razón de riesgos, fue de 7,69, una indicación de que sería mucho más probable que una paciente que presenta DG presente SM al término del puerperio.

Encontramos diferencias altamente significativas $(p<0,01)$ entre los promedios de ambos grupos para las variables IMC puérpera, obesidad abdominal, presión arterial diastólica, IMC madre. En el resto de las variables no se encontraron diferencias significativas entre los promedios de los grupos control y estudio.

\section{Tabla 7. Tabla de contingencia para diagnóstico de síndrome metabólico para los grupos estudio y control}

\begin{tabular}{|lccc|}
\hline Frecuencia & $\begin{array}{c}\text { Con } \\
\text { diagnóstico } \\
\text { SM }\end{array}$ & $\begin{array}{c}\text { Sin } \\
\text { diagnóstico } \\
\text { SM }\end{array}$ & Total \\
\hline Estudio & 32 & 26 & 58 \\
Control & 8 & 50 & 58 \\
Total & 40 & 76 & 116 \\
\hline
\end{tabular}

$\mathrm{CHI}^{2}: 21,979$ significativo $(\mathrm{p}<0,01)$. 


\section{DisCUSIÓN}

Las diferencias de los promedios de peso materno, el IMC y obesidad abdominal encontrados, coinciden con los hallazgos de Despres ${ }^{14}$, quien reconoce el sobrepeso y la obesidad como responsables del riesgo vascular y del exceso de mortalidad por enfermedades cardiovasculares, además reporta el incremento de la obesidad abdominal como componente clave de la insulinorresistencia.

La frecuencia levemente elevada, aunque no significativa, de hipertensión en las mujeres con DG de este estudio, concordaría con el estado de insulinorresistencia gatillado durante el embarazo y apoya la idea de DG como modelo de diabetes mellitus tipo 2 planteada por Cubeddu ${ }^{15}$.

Igualmente, los resultados de las comparaciones del IMC promedio del padre y de la madre, así como los antecedentes de tabaquismo paterno, la proporción de pacientes con antecedentes familiares de síndrome hipertensivo del embarazo (SHE) y de diabetes, tienen relación con lo expuesto por Poulsen y $\mathrm{Vaag}^{1}$, quien estudió el impacto relativo de estos factores en el desarrollo del SM y DG.

Molina et $\mathrm{al}^{16}$ determinaron los niveles de colesterol total, LDL, HDL, triglicéridos y apolipoproteínas AI y B en sangre del cordón de RN con restricción del crecimiento intrauterino (RCIU), tanto simétricos como asimétricos y los compararon con los de un grupo de RN de término normal, observando que existían diferencias en el comportamiento de dichos parámetros, especialmente en los triglicéridos los cuales estaban elevados. Así también, Yajnik et $\mathrm{al}^{13}$ mencionan que existen evidencias emergentes de que el deterioro en la vasodilatación dependiente del endotelio está presente en poblaciones con futuro riesgo de diabetes y aún en niños con bajo peso al nacer, quienes podrían presentar elementos del SM más tarde en la vida. Ambos hallazgos muestran relación con los resultados obtenidos en esta investigación, en la cual la condición PEG tuvo una frecuencia significativamente superior en el grupo estudio, con una RR próxima a 20, lo que indica, que efectivamente la condición PEG al nacer favorece el desarrollo de DG.

La elevación de la glicemia y los triglicéridos, y el menor HDL del grupo estudio comparado con el grupo control, son similares a los observados por Hanson (2000).

Griffin et $\mathrm{al}^{17}$ y Jensen et $\mathrm{al}^{9}$, estudiaron mujeres con DG comparándolas con un grupo control, en las cuales midieron un perfil de riesgo materno que consistió en glicemia ayunas y post prandial, triglicéridos, colesterol HDL, presión arterial e IMC, presentando las pacientes con DG un riesgo materno aumentado para SM, lo que coincide con los resultados de éste estudio.

Griffin et $\mathrm{al}^{17}$ realizaron un screening para DG, incorporando medición de glicemias, triglicéridos, insulinemia, encontrando relación significativa de los niveles séricos aumentados, lo que está relacionado con la frecuencia de diagnóstico de SM encontrado en el grupo estudio de esta investigación en la cual no se midió insulinemia.

La proporción de pacientes con SM y con DG observadas en este estudio, concuerda con la prevalencia señalada por la OMS en EEUU, señalando que la DG se considera precursora de la diabetes mellitus tipo 2 (DM2), según lo señalado por Masuzaki y Paterson ${ }^{8}$.

Los niveles de presión arterial, talla, peso y circunferencia de cintura de las puérperas de 42 días post parto, en conjunto el IMC de puérperas, su condición al nacer, más los antecedentes familiares de las puérperas de hipertensión arterial, de diabetes mellitus, así como el peso, talla e IMC de los padres de la puérpera y los hábitos de los padres de éstas, predisponen a éstas mujeres a presentar un riesgo de enfermedad cardiovascular.

Los resultados obtenidos de niveles séricos de triglicéridos, glicemia, HDL, en el grupo de estudio, concuerdan con niveles requeridos para realizar el diagnóstico de SM, siendo posible determinar que es aproximadamente 8 veces más probable $(\mathrm{RR}=7,69)$, que una paciente que presenta DG desarrolle también SM al término del puerperio, comparada con pacientes que no presentan DG (grupo control). Esto permite concluir que existe una fuerte relación entre diabetes gestacional y síndrome metabólico.

Consideramos importante, por tanto, promover desde todos los sectores involucrados en el crecimiento y desarrollo del niño, hábitos de vida saludables que se traduzcan en cambios positivos desde edades tempranas y que se prolonguen a lo largo de las diferentes etapas de la vida. Para ello 
se debe promover el estilo de vida saludable, es decir un cambio conductual que contemple una alimentación equilibrada, actividad física regular y control clínico precoz de la enfermedad.

Los resultados obtenidos nos permiten reflexionar sobre el rol de la DG en la patogénesis del SM, ya que la conjugación de los factores de riesgo a los que está expuesta la embarazada con DG las deja en una situación de riesgo y vulnera-

\section{REFERENCIAS}

1. Pulsen P, VaAg A. Genetic versus environmental etiology of the metabolic Syndrome among male and female twins. Diabetologia 2001; 44: $537-43$.

2. National Cholesterol Education Program (NCEP). Executive Summary of the Third Report of the National Cholesterol Education Program. Expert panel on detection, evaluation and treatment of high blood cholesterol in adults (Adult Treatment Panel III). JAMA 2001; 285: 2486-500.

3. Hanson RL. Evaluation of simple indices of insulin sensitivity and insulin secretion for use in epidemiologic studies. Am J Epidemiol 2000; 151: 190-8.

4. Ford ES, Giles WH, Dietz WH. Prevalence of the Metabolic Syndrome among US Adults: Findings From the Third National Health and Nutrition Examination Survey. JAMA 2002; 16: 356-9.

5. MINSAL, Encuesta Nacional de Salud 2003: Desarrollo del trabajo de campo.

6. Lebovitz HF. Insulin resistance: definition and consequences. Exp Clin End Diabetes 2001; 109 Suppl 2: 135-48.

7. CEDIP. Guias clinicas peri natales 2005; 16: 21923.

8. Masuzaki H, Paterson J. A transgenic model of visceral obesity and the metabolic syndrome. Science 2001; 294: 2166-70.

9. Jensen DM, Sorensen B, Feilberg-Jorgensen N, WesteRgaARD JG, BECK-NIELSEN H. Maternal and perinatal outcomes in 143 Danish women with gestational bilidad, necesaria de atender en futuras investigaciones.

Por último, destacamos la importancia de realizar en todas las mujeres con diabetes gestacional, y particularmente en aquella con diagnóstico de síndrome metabólico al finalizar su puerperio, seguimiento, de manera de detectar precozmente el desarrollo de diabetes, e intervenir en la disminución de su riesgo global.

diabetes mellitus and 143 controls with a similar risk profile. Diabet Med 2000; 17: 281-6.

10. Isomaa $B$, Henricsson M. The metabolic syndrome influences the risk of chronic complications in patients with II diabetes. Diabetología 2001; 44: 1148-54.

11. Tiikrainen M, Marjo T, Annamaija H, et al. Liver-fat accumulation and insulin resistance in obese women with previous gestational diabetes. Obesity Research 2002; 10: 859-67.

12. Poulter NR. Birthweights, maternal cardiovascular events and Barker hypothesis. Lancet 2001; 357: 1990-1.

13. Yajnik CS, fall CH, Coyaji KJ, Hirve SS, Rao S, Barker DJ et aL. Neonatal anthropometry: the thin-fat Indian baby. The Pune Maternal Nutrition Study. Int J Obes Relat Metab Disord 2003; 27: $173-80$

14. Despres JP. Health consequences of visceral obesity. Ann Med 2001; 3: 534-41.

15. CuBEDDu LX. Insulin resistance and upper - normal glucose levels in hypertension: a review. $J$ Human Hypertens 2002; 16 Suppl 1: S 52-55.

16. Molina M, Casanueva E, Cid X, Ferrada MC, Pérez $\mathrm{R}$, Dios G et al. Perfil lipídico en recién nacidos con retardo del crecimiento intrauterino. Rev Méd Chile 2000; 128: 741-8.

17. Griffin ME, Coffey M, Johnsont H, Scanlon P, Foley M, STRONGet J et al. Universal vs. risk factor-based screening for gestational diabetes mellitus: detection rates, gestation at diagnosis and outcome. Diabet Med 2000; 17: 26-32. 